\section{Loeys-Dietz syndrome is a specific phenotype and not a concomitant of any mutation in a gene involved in TGF- $\beta$ signaling}

To the Editor: The authors of the "narrative review" of LoeysDietz syndrome (LDS), "Loeys-Dietz Syndrome: A Primer for Diagnosis and Management," published in this issue, propose a new nosology for LDS based on genotype and provide their perspectives on the pleiotropic phenotype and management of all affected organ systems. ${ }^{1}$ TGFBR2 mutations were initially described in patients diagnosed with Marfan syndrome. ${ }^{2}$ In 2005, Loeys and colleagues ${ }^{3}$ described severely affected patients with aggressive aortic disease with dissections with minimal enlargement of the aortic root and diffuse arterial tortuosity, coupled with other clinical features (bifid uvula, craniosynostosis, hypertelorism, and clubfoot) due to mutations in TGFBR1 and TGFBR2. This phenotype was labeled LDS. Subsequently, patients with thoracic aortic disease with minimal or no craniofacial dysmorphism were also found to have mutations in TGFBR1 and TGFBR2, as well as in SMAD3 and TGFB2, other genes encoding proteins in the TGF- $\beta$ pathway. MacCarrick et al. ${ }^{1}$ propose to define any patient with a pathogenic variation in any of these four TGF- $\beta$ pathway genes as having LDS. Furthermore, they suggest that any patient with a mutation in any of these four genes should undergo valvesparing aortic root surgery with minimal enlargement, criteria previously reserved for patients with the severe LDS phenotype with mutations in TGFBR1 and TGFBR2. The opinion that anyone with a pathogenic variation in any of these four genes qualifies for a diagnosis of LDS has been codified in Online Mendelian Inheritance in Man (http://omim.org/).

In our collective published and unpublished experience, diagnosing and managing all patients with mutations in these genes similarly is inappropriate. Many patients with mutations in TGFBR1, TGFBR2, SMAD3, and TGFB2 do not have a phenotype consistent with LDS nor do they have an aggressive arterial disease., ${ }^{4,5}$ Although arterial involvement beyond the aortic root is established for TGFBR1, TGFBR2, and $S M A D 3$, there is no evidence that TGFB2 mutations cause arterial disease to the same extent. Osteoarthritis and neurological features can be associated with mutations in SMAD3 but not with the other genes. The clinical picture also differs considerably between those with TGFBR1 and TGFBR2 mutations. ${ }^{5}$ Moreover, some mutations in genes involved in the TGF- $\beta$ pathway do not lead to aortic disease (e.g.,
Camurati-Engelmann disease with mutations in TGFB1). Arterial tortuosity, which has been proposed to be diagnostic for LDS, is observed in patients with mutations in other genes, such as FBN1, FBNL4, PRKG1, and SLC2A10.

Therefore, labeling mutations in all these genes as diagnostic for LDS risks improper management (limitations of exercise, insurance and employment, unnecessary medication, and prophylactic surgery with only mild aortic dilatation), inaccurate reproductive counseling, and psychosocial distress. Furthermore, MacCarrick et al. ${ }^{1}$ suggest high-dose angiotensin receptor blockade in all patients, evidence for which is entirely lacking in LDS and still emerging for Marfan syndrome. Finally, labeling a phenotype based solely on mutations in specific genes sets a precedent that conflicts with experience with other Mendelian conditions. To cite but two examples, mutations in FBN1 cause not only Marfan syndrome but at least eight other conditions, some completely distinct from Marfan syndrome (e.g., geleophysic dysplasia, Weill-Marchesani syndrome), and mutations in TGFBR1 cause multiple self-healing squamous epithelioma syndrome, a phenotype completely distinct from LDS. In addition to such intragenic variability, we are well aware of the possibility of intrafamilial variability, which emphasizes the utility of biomarkers that might help tailor therapy and indications for vascular surgery. Clinical features of LDS and arterial tortuosity may well be such markers. ${ }^{6}$

We recently proposed an alternative classification scheme for heritable thoracic aortic diseases. ${ }^{7}$ This nosology is based on the underlying gene but recognizes that diverse aortic phenotypes can arise from the same locus. In addition to classic heritable disorders of connective tissue (e.g., vascular EhlersDanlos syndrome, Marfan syndrome) and heritable thoracic aortic diseases due to mutations in genes for proteins involved in smooth muscle contraction (e.g., ACTA2, MYH11, MYLK, PRKG1), another category is genes specifying components of the TGF- $\beta$ signaling pathway (e.g., TGFBR1, TGFBR 2, TGFB2, SMAD3). This latter category also encompasses a variety of heritable thoracic aortic diseases, including LDS.

As MacCarrick et al. ${ }^{1}$ note, they have published a "narrative review," which is "based on a systematic literature review, expert opinion, and standard-of-care practices from the center with the largest patient population of these disorders in the world." If "expert opinion" is drawn principally from a center (albeit a great academic one) to which are referred the most severe and difficult cases, then the perspectives of those experts may be biased. Gene-specific clinical data from multiple institutions should be collected and analyzed to define vascular treatment guidelines for all heritable thoracic aortic disease genes. Based on our experience with diagnosing, counseling, and caring for patients and families with aortic diseases from general international populations, we respectfully disagree with lumping together as having LDS all people with mutations in TGFBR1, TGFBR2, SMAD3, 
and TGFB2, along with "other TGF- $\beta$ signaling pathway genes yet to be associated with disease" for which the clinical characteristics have yet to be defined.

\section{DISCLOSURE}

The authors declare no conflict of interest.

Reed Pyeritz, $M D, P h D^{1}$, Guillaume Jondeau, $M D, P h D^{2}$, Rocio Moran, $M D^{3}$, Julie De Backer, $M D, P h D^{4}$, Eloisa Arbustini, $M D^{5}$, Anne De Paepe, $M D, P h D^{4}$ and Dianna Milewicz, $M D, P h D^{6}$; for the Montalcino Aortic Consortium

${ }^{1}$ Perelman School of Medicine at the University of Pennsylvania, Philadelphia, Pennsylvania, USA $;{ }^{2}$ AP-HP Hôpital Bichat, INSERM, Paris, France; ${ }^{3}$ Cleveland Clinic, Cleveland, Ohio, USA; ${ }^{4}$ University of Gent, Gent, Belgium; ${ }^{5}$ IRCCS Fondazione Policlinico San Matteo, Pavia, Italy; ${ }^{6}$ University of Texas Health Science Center at Houston, Houston, Texas, USA. Correspondence: Reed Pyeritz (reed.pyeritz@uphs.upenn.edu)

\section{Response to Pyeritz et al.}

To the Editor: Subsequent to the meeting of the "Montalcino Consortium," the Marfan Foundation convened a more inclusive gathering in Boston of all invested parties to discuss the important issue of nosology for inherited presentations of thoracic aortic aneurysm. In addition to representatives from the Montalcino Consortium, participants included leaders from many additional centers of excellence in the care of thoracic aortic disease and patients and representatives from patient advocacy groups including the US and Canadian Marfan foundations and the US Loeys-Dietz Syndrome Foundation. Virtually all of the comments and concerns raised by Pyeritz et al. ${ }^{1}$ in their letter, "Loeys-Dietz Syndrome Is a Specific Phenotype and Not a Concomitant of Any Mutation in a Gene Involved in TGF- $\beta$ Signaling," were discussed in detail. We welcome this opportunity to more thoroughly present the prevailing themes of this meeting.

There was uniform consensus that knowledge about the gene or specific mutation underlying a disease can serve as a proxy for the biochemical and cellular events that drive disease initiation or progression and that influence response to therapy. This knowledge often provides predictive value with regard to when and where disease will manifest; the character of the disease (e.g., mild or severe, and more or less predictable than average); the best diagnostic, follow-up, and therapeutic protocols to apply; and the spectrum of risks for family members (present and future). The ability to "bin" patients into a specific predisposition class (based on commonality of phenotype/genotype/mechanism) increases the potential for anticipatory counseling and management while minimizing bias or other confounding factors. Such principles underlie current practices in the diagnosis and management of Marfan syndrome (MFS), in which the presence of a pathogenic FBN1 mutation or an unequivocally affected family member, in combination with aortic root enlargement, is sufficient for the

\section{REFERENCES}

1. MacCarrick G, Black JH III, Bowdin S, et al. Loeys-Dietz syndrome: a primer for diagnosis and management. Genet Med 2014;16:576-587.

2. Mizuguchi T, Collod-Beroud G, Akiyama T, et al. Heterozygous TGFBR2 mutations in Marfan syndrome. Nat Genet 2004;36:855-860.

3. Loeys BL, Chen J, Neptune BR, et al. A syndrome of altered cardiovascular, crainiofacial, neurocognitive and skeletal development caused by mutations in TGFBR1 or TGFBR2. Nat Genet 2005;37:275-281.

4. Attias D, Stheneur C, Roy C, et al. Comparison of clinical presentations and outcomes between patients with TGFBR2 and FBN1 mutations in Marfan syndrome and related disorders. Circulation 2009;120: 2541-2549.

5. Tran-Fadulu V, Pannu H, Kim DH, et al. Analysis of multigenerational families with thoracic aortic aneurysms and dissections due to TGFBR1 or TGFBR2 mutations. J Med Genet 2009;46:607-613.

6. Morris SA, Orbach DB, Geva T, et al. Increased vertebral artery tortuosity index is associated with adverse outcomes in children and young adults with connective tissue disorders. Circulation 2011;124:388-396.

7. Pyeritz RE. Heritable thoracic aortic disorders. Curr Opin Cardio/ 2014;29:97-102.

doi:10.1038/gim.2014.63

diagnosis of MFS irrespective of the presence and/or severity of other systemic findings, ranging from catastrophic infantile presentations that often associate with striking dysmorphism to exceedingly mild adult presentations in individuals who do not show outward features of MFS. This alerts caregivers with variable familiarity with the intricacies of diagnosis to the spectrum of possibilities and informs patient management (e.g., the need for ophthalmologic evaluations, proper imaging modalities, and surgical thresholds). A description of these widely endorsed and applied priorities and nosologic practices included the leadership of the Montalcino Consortium as authors, and similar practices are in place (and productive) for other presentations of aneurysm, prominently including vascular Ehlers-Danlos syndrome., ${ }^{2,3}$

The term "Loeys-Dietz syndrome" (LDS) was initially applied to describe a condition, caused by mutations in either of the genes that encode subunits of the transforming growth factor (TGF)- $\beta$ receptor (TGFBR1 or TGFBR2), that associates many features of MFS (arachnodactyly, pectus deformity, scoliosis, dural ectasia, and aortic root aneurysm) with other discriminating features in the craniofacial (hypertelorism, cleft palate, bifid uvula, and craniosynostosis), skeletal (cervical spine malformation and/or instability and clubfoot deformity), and cutaneous (translucent skin, easy bruising, and dystrophic scars) systems. ${ }^{4}$ Most importantly, patients with LDS often show a widespread and aggressive vasculopathy (arterial tortuosity, aneurysms throughout the arterial tree, dissections at young ages and at relatively small vascular dimensions that do not infer risk or provoke surgery in MFS, and many other conditions). Within 1 year (in 2006), a second publication expanded the phenotypic spectrum of LDS to include individuals with TGFBR1 or TGFBR2 mutations with a similarly diffuse and aggressive vascular phenotype but only subtle or even absent craniofacial and/or skeletal manifestations. ${ }^{5}$ Around the same time, others proposed use of the diagnosis of familial thoracic aortic aneurysm and dissection for such patients, a term previously applied for patients with mutations in genes encoding components of 